\title{
The 'Real World' Clinic Measured Visual Acuity Versus the Best Correct Visual Acuity Measurement in Patients Treated for Neovascular Age-Related Macular Degeneration
}

Obaid Kousha ( $\square$ ok32@st-andrews.ac.uk)

University of St Andrews

Amritpal Chaggar

University Hospitals Coventry and Warwickshire

Sergio Pagliarini

University Hospitals Coventry and Warwickshire

\section{Research Article}

Keywords: Visual acuity, Age-related macular degeneration, Accuracy, Precision

Posted Date: September 23rd, 2021

DOI: https://doi.org/10.21203/rs.3.rs-892155/v1

License: (c) (1) This work is licensed under a Creative Commons Attribution 4.0 International License.

Read Full License 


\section{Abstract}

\section{BACKGROUND}

In ophthalmology clinics, the visual acuity (VA) is usually measured by non-refracting healthcare professionals (HCPs). We compared the 'real world' or clinic measured VA versus the best-corrected visual acuity measurement in patients with neovascular age-related macular degeneration (nAMD).

\section{METHODS}

During the same clinical session using Early Treatment of Diabetic Retinopathy Study (ETDRS) vision chart, monocular distance VA was measured by non-refracting HCPs in nAMD patients and compared to the monocular distance VA measured by an optometrist with subjective refractive correction (BCVA). The study was powered to detect a difference of $>5$ ETDRS letters between the groups and for HCP measured VA to detect a BCVA between 6/12 and 6/96 (National Institute of Health and Care Excellence condition of approval of nAMD treatment with licensed drugs in the UK).

\section{RESULTS}

Data from 167 patients (324 eyes) were analysed. Absolute mean difference in BCVA versus HCP measured VA was 5.71 (95\% confidence interval (Cl) 5.10-6.41) ETDRS letters ( $p$-value<0.001). Using Bland-Altman method, limits of agreement between the two groups was found to be between 18.39 and -14.66 ETDRS letters. The prevalence of BCVA between $6 / 12$ and $6 / 96$ was $53.40 \%(95 \% \mathrm{Cl} 47.96$ $58.83 \%)$. In detecting this BCVA bracket, the non-refracting HCP measured VA had a sensitivity of $91.91 \%$ (95\% Cl 87.84-95.97\%) and a specificity of $84.77 \%$ (95\% Cl 79.04-90.50\%).

\section{CONCLUSIONS}

The 'real world' HCP measured VA was found to have considerable inaccuracy and imprecision when compared to BCVA in nAMD patients. Unreliable VA measurement can have important implications in assessing and treating eye conditions, including nAMD.

\section{Background}

The most common method of determining the level of vision is to measure monocular distance visual acuity. Monocular distance visual acuity (VA) is the minimum angle of resolution of two points achieved by the visual system under standard conditions (1). It is commonly measured as the number of highcontrast letters, which progressively reduce in size, that a person is able to read from a standardised chart at a pre-defined distance. It is a relatively simple and quick way to quantify the level of vision in a patient in both the clinical and research environment, including patients with age-related macular degeneration.

Age related macular degeneration (AMD) is a disease affecting the central area of the retina. This results in loss of central vision and deterioration of the patient's ability to undertake tasks such as reading, 
recognise faces, drive a vehicle, with a significant reduction in the quality of life (2). It is an agingassociated disease and approximately $5 \%$ of the people aged 65 and over in the United Kingdom (UK) are affected by the advanced form of the disease (3). Indeed, in England \& Wales, over 30000 people are registered as partially sighted or blind annually, half of whom are due to AMD (4). Neovascular, wet or exudative AMD (nAMD) is the late stage manifestation of AMD more frequently associated with devastating loss of central vision (5).

VA measurement is an important tool for the assessment and management of patients with nAMD (6). Ranibizumab and Aflibercept are the two licensed drugs approved by the National Institute for Health and Care Excellence (NICE) to treat nAMD in England $(7,8)$. A key NICE condition of approval of nAMD treatment with licensed drugs is for the VA in the patient's affected eye(s) to be between 6/12 and 6/96 on a Snellen chart. This equates to a VA score between 24 and 73 letters on the ETDRS vision chart. VA measurement is also the primary outcome of all clinical trials on the treatment of nAMD.(2) Changes in VA are used to measure response to treatment (9). Notably, the loss or gain of three or six lines of vision on the ETDRS chart defines respectively the outcome of moderate or severe clinically significant visual loss or gain (9). In the UK, VA is used to determine the level of visual impairment (10). Patients with full visual fields may be registered as severely or partially visually impaired if the VA in the better eye is less than 24 ETDRS letters ( $3 / 60$ on Snellen chart) or between 24 and 38 letters ( $3 / 60$ to 6/60 Snellen) respectively (10). Conversely, one of the main criteria to drive a car in the UK is a binocular VA of at least $6 / 12$ on the Snellen chart or 70 letters on ETDRS chart (11).

The reference or "gold" standard measurement of the VA is the best corrected VA (BCVA). In this work, we define BCVA as monocular VA measured after correction of the refractive error under standardised conditions, which is usually performed by an optometrist. However, measuring BCVA in every patient is very resource-intensive, hence not practical in a busy eye clinic. Therefore, the VA in patients with nAMD is usually measured by the non-refracting allied health care professionals (HCPs). HCPs include nurses, technicians and healthcare assistants with a variety of ophthalmic training background. When measuring VA, HCPs are not expected to carry out subjective refraction.

Anecdotal evidence and our clinical experience suggest that on occasions the VA measured by a nonrefracting HCP may differ significantly from the BCVA. The magnitude of such discrepancy has both clinical and legal implications.

Furthermore, inter-centre variation in nAMD VA outcomes, not fully explained by patient or treatment characteristics, has emerged in the UK (12). Reliability of the "real world" VA outcomes depends on the quality of VA measurements.

This report is the first to ascertain the accuracy and precision of non-refracting HCP measured VA to BCVA in nAMD patients.

\section{Method}




\section{Aims:}

Primary outcomes:

- To ascertain if there is a difference of $>5$ ETDRS letters between optometrist measured BCVA and VA measured by non-refracting HCP, which we will refer to as BCVA versus VA

- To determine the sensitivity and specificity of the non-refracting HCP measured VA for identifying patients with a BCVA between 24 and 73 ETDRS letters (The visual acuity bracket in the UK to be eligible to NICE approved anti-VEGF treatment in nAMD)

Secondary outcomes:

- To determine the sensitivity and specificity of the non-refracting HCP measured VA for identifying patients with a BCVA of < 70 ETDRS letters (Visual acuity not eligible for driving in the UK)

- To determine the sensitivity and specificity of the HCP measured VA for identifying patients with a BCVA of < 24 ETDRS letters (VA compatible with severe visual impairment registration)

\section{Population:}

We aimed to recruit patients, suffering from nAMD in at least one eye, attending the macula clinic for assessment or treatment. The study was carried out between 3rd March 2019 and 31st January 2020 in the Macula Clinic, Hospital of St Cross, University Hospitals Coventry and Warwickshire NHS Trust, Rugby, UK.

\section{Visual acuity measurement:}

For the purpose of this study, the VA was measured prior to any assessment or treatment (13). The VA was measured using the $4 \mathrm{~m}$ LogMAR ETDRS original charts 1, 2 and R. The charts were mounted on a Precision Vision LogMAR lightbox and retro-illumination provided by two internal daylight 20-watt fluorescent tubes (burned in for 100 hours, prior to use in the clinics). The room lighting was standardised. The VA assessment was undertaken (i.e. the non-refracting HCP or optometrist) in no particular order. Both the non-refracting HCP and the optometrist were blinded from each other's measurements. All the non-refracting HCPs were trained to carry out visual acuity assessment.

The recommended method for visual acuity assessment was as follows:

I. Each eye was tested separately to obtain monocular visual acuity

II. The patient was seated at 4 metres from the chart and instructed to read the smallest line of letters visible

III. The patient was required to correctly identify at least 3 letters on a line for testing to continue

IV. If $<3$ letters were correctly identified on a line, the patient was instructed to read the line above to confirm at least 3 letters were read correctly and this process was repeated until at least 3 letters were correctly identified on a line 
V. Following this the patient was instructed to continuing reading the lines below until at least 3 letters were identified correctly on a line.

VI. Eccentric fixation was encouraged and testing stopped when the patient was only able to identify $<3$ letters on a line

VII. If $<3$ letters from the top line were read at $4 \mathrm{~m}$, the testing distance was to be reduced to $1 \mathrm{~m}$ and testing was performed as detailed in III-VI above

Over the period of study, 10 different non-refracting HCPs were involved. To carry out VA assessment, the non-refracting HCPs instructed patients to wear their distance spectacles if they were in possession and a pinhole test was also used. The standard non-refracting HCP practice was to use one ETDRS chart for both eyes. The best measured VA (with or without pinhole), by the HCP, was used for analysis.

The best corrected visual acuity was also measured by a qualified clinical trial optometrist (AC) to determine subjective refractive error and record the BCVA utilising a separate chart for each eye.

\section{Sample size:}

The sample size was calculated based on the result of a pilot study $(n=54)$, our clinical experience and after discussion with a medical statistician.

Power calculation was carried out using statistical software G*Power 3.1.9.4 for Windows (14).

- $\mathrm{N}$ for a difference of $\geq 5$ ETDRS letters between BCVA and HCP measured VA with an effect size = 0.40 , Power of $99.9 \%$, and two-tailed type II error of $0.001=260$ (calculated for $t$-test comparing two dependent means (matched pairs))

- $\mathrm{N}$ for a specificity of $90 \%$ with a two-sided confidence interval of $95 \%$ for the VA as measured by the non-refracting HCP for identifying patients with a BCVA of 24 to 73 ETDRS letters with a prevalence of a BCVA between 24 and 73 ETDRS letters of $45 \%=251$

- $\mathrm{N}$ for a sensitivity of $90 \%$ with a two- sided confidence interval of $95 \%$ for the VA as measured by the non-refraction HCP for identifying patients with a BCVA of 24 to 73 ETDRS letters with a prevalence of a BCVA between 24 and 73 ETDRS letters of $45 \%=307$

- Therefore $\mathrm{N}=307$ eyes was chosen

\section{Data analysis:}

The data was collated in a spreadsheet and analysed using IBM SPSS Statistics for Windows, Version 22. The tests were two tailed and type 1 error was set at $a=0.05$. Student's $t$ test was used to compare the two dependent variable means (matched pairs) of continuous data. Bland-Altman plot was used to analyse the two methods of VA measurement (15). Pearson correlation was used to test correlation between continuous data.

\section{Results}


In this cross-sectional study we recruited 167 patients (324 eyes). The mean BCVA was 56.76 ETDRS letters with standard deviation of 25.23 ETDRS letters.

The absolute mean difference in BCVA versus non-refracting HCP measured VA was 5.71 (95\% confidence interval $(\mathrm{Cl}) 5.10-6.41)$ ETDRS letters ( $p$-value< 0.001$)$. In 114 eyes (35.19\%) there was more than 5 ETDRS letters difference between the BCVA and the non-refracting HCP measured VA. When compared to BCVA, the non-refracting HCP measured VA overestimated vision by 5 ETDRS letters in 38 eyes $(11.73 \%)$ and under estimated vision by 5 ETDRS letters in 76 eyes $(23.46 \%)$.

However, as the Bland-Altman plot (Fig. 1) demonstrates, there is variation in the difference between BCVA and non-refracting HCP measured VA with limits of agreement between 18.39 ETDRS letters and 14.66 ETDRS letters. Pearson correlation revealed a negligible correlation between the BCVA and the BCVA minus non-refracting HCP measured VA $(r=0.17, p$-value $=0.002)$, indicating there are no systematic under reporting of the VA by the non-refracting HCPs at one extreme of the BCVA compared to the other. However, the Pearson correlation between the BCVA and the absolute value of the BCVA minus non-refracting HCP measured VA showed a weak negative correlation $(r=-0.323, p$-value $<0.001)$, suggesting a larger deviation in non-refracting HCP measured VA from the BCVA at lower levels of the BCVA.

The prevalence of eyes with BCVA between 24 and 73 ETDRS letters bracket was $53.40 \%$ (95\% Cl 47.96$58.83 \%$ ). In detecting this BCVA bracket, the non-refracting HCP measured VA had a sensitivity of $91.91 \%$ (95\% Cl $87.84-95.97 \%)$, a specificity of $84.77 \%$ (95\% Cl 79.04-90.50\%), a positive predictive value of $87.36 \%(95 \% \mathrm{Cl} 82.54-92.19 \%)$ and a negative predictive value of $90.14 \%$ (95\% $\mathrm{Cl} 85.24-95.04 \%)$ (Table 1) 
Table 1

Sensitivity, specificity, positive and negative predictive value of non-refracting HCP measured VA in detecting BCVA $=24-73$ ETDRS letters

\begin{tabular}{|lllll|}
\hline & \multicolumn{4}{c|}{ BCVA= 24-73 ETDRS letters } \\
\hline $\begin{array}{l}\text { Non-refracting HCP measured VA }=24- \\
\text { 73 ETDRS letters }\end{array}$ & Yes & 159 & No & Total \\
\cline { 2 - 5 } & No & 14 & 128 & 182 \\
\hline Sensitivity & Total & 173 & 151 & 324 \\
\hline Specificity & $91.91 \%(87.84-95.97 \%)+$ & \\
\hline Positive predictive value & $84.77 \%(79.04-90.50 \%)+$ & \\
\hline Negative predictive value & $87.36 \%(82.54-92.19 \%)+$ & \\
\hline Prevalence & $90.14 \%(85.24-95.04 \%)+$ & \\
\hline † = 95\% Confidence interval & $53.4 \%(47.96-58.83 \%)+$ & \\
\hline
\end{tabular}

The prevalence of eyes with BCVA $<70$ ETDRS letters was 59.26\% (95\% Cl 53.91-64.61\%). In detecting a BCVA of $<70$ ETDRS letters, the non-refracting HCP measured VA had a sensitivity of $96.88 \%(95 \% \mathrm{Cl}$ 94.17-99.34\%), a specificity of $88.64 \%$ (95\% Cl 83.22-94.05\%), a positive predictive value of $92.44 \%$ (95\% Cl 88.90-96.17\%) and a negative predictive value of 95.12\% (95\% Cl 92.31-98.93\%) (Table 2). 
Table 2

Sensitivity, specificity, positive and negative predictive value of non-refracting HCP measured VA in detecting BCVA $<70$ ETDRS letters

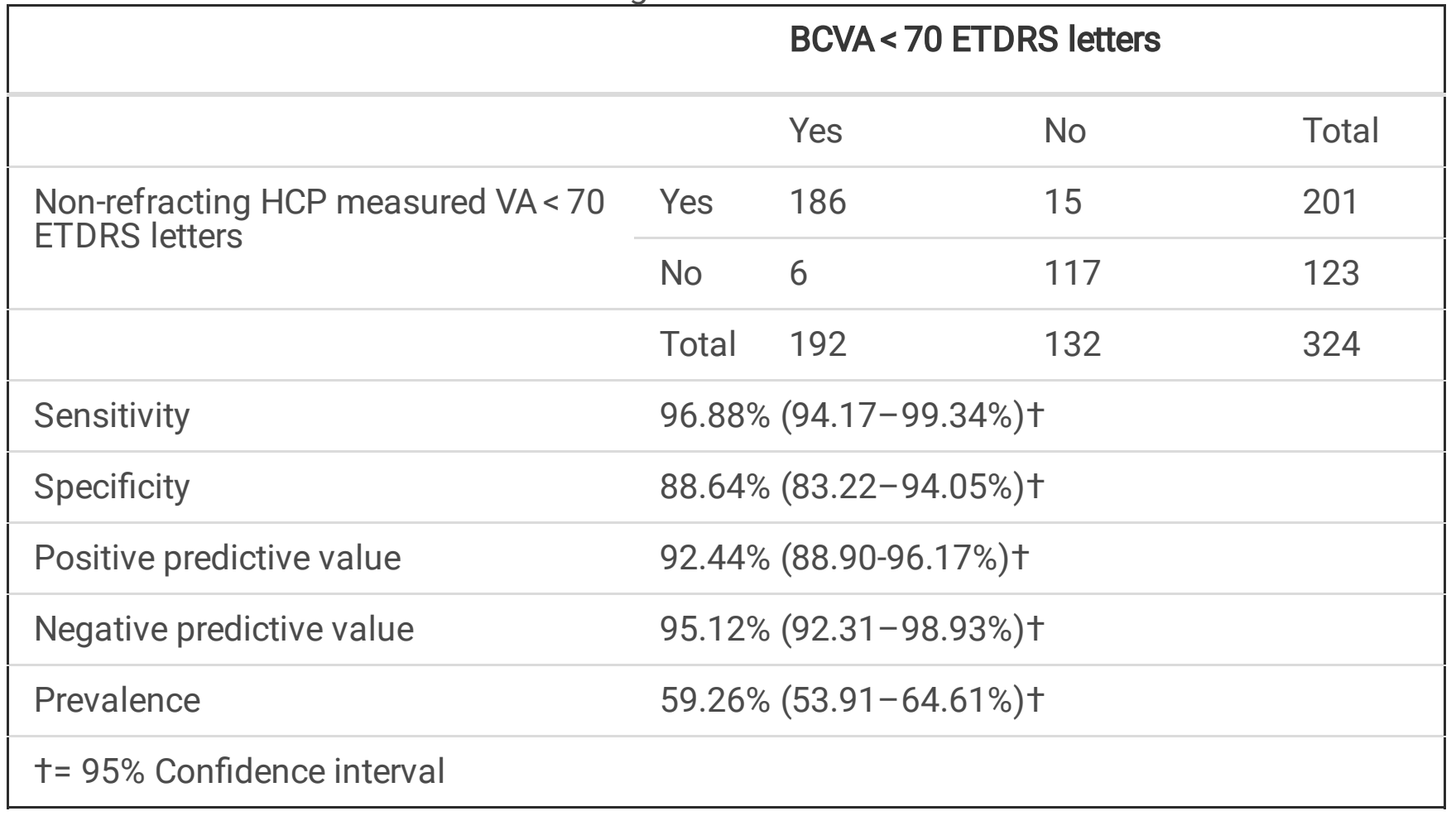

The prevalence of eyes with BCVA< 24 ETDRS letters was 13.27\% (95\% Cl 9.58-16.97\%). In detecting a BCVA of $<24$ ETDRS letters, the non-refracting HCP measured VA had a sensitivity of $76.74 \%(95 \% \mathrm{Cl}$ $64.12-89.37 \%$ ), a specificity of $97.86 \%$ (95\% Cl $96.17-99.56 \%$ ), a positive predictive value of $84.62 \%$ (95\% Cl 73.29-95.94\%) and a negative predictive value of 96.49\% (95\% Cl 94.36-98.63\%) (Table 3). 
Table 3

Sensitivity, specificity, positive and negative predictive value of non-refracting HCP measured VA in detecting BCVA < 24 ETDRS letters

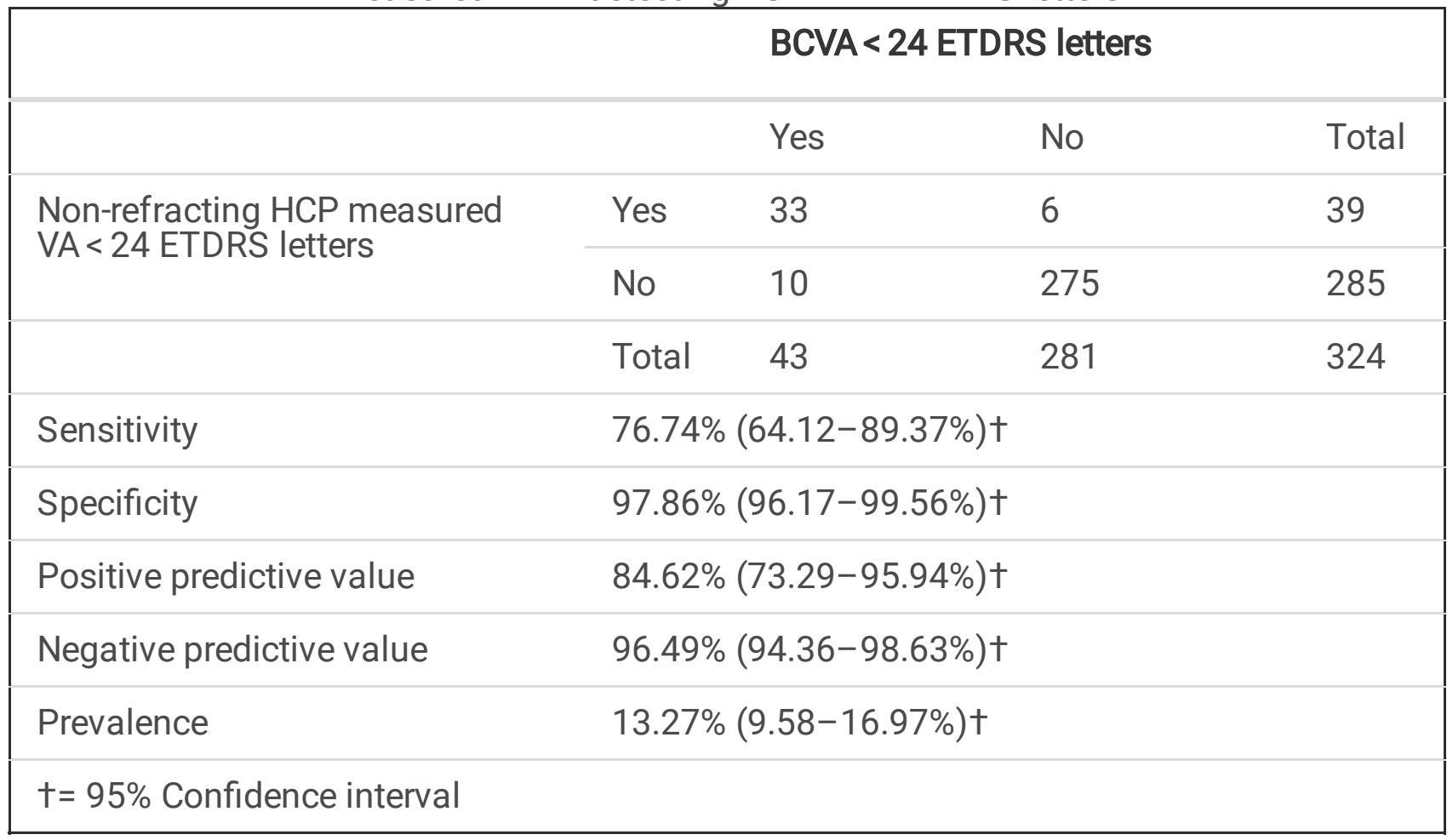

\section{Discussion}

In the UK, visual acuity is measured by uncertified non-refracting HCP staff in eye clinics. This is the first study to comprehensively investigate and demonstrate the limitations in non-refracting HCP measured VA when compared to the best corrected visual acuity as measured by an optometrist with subjective refractive error correction on the same day.

The non-refracting HCP measured VA was > 5 ETDRS letters inaccurate in a third of the patients. The limits of agreement on Bland-Altman plot indicates that the non-refracting HCP measured VA is imprecise, measuring VA from 18.39 ETDRS letters higher to -14.66 ETDRS lower than the BCVA. This inaccuracy and imprecision in the non-refracting HCP measured VA may have clinical implications when assessing and managing patients with eye conditions. In our example of AMD patients, a variation of less than \pm 5 ETDRS letters in between visits in eyes with nAMD is considered to be clinically non-significant, while a loss of $>5$ letters is deemed as potentially clinically significant and an improvement of $\geq 5$ ETDRS letters is considered as a 'good' response to treatment with the licensed anti-VEGF drugs (9). The seminal clinical trials providing evidence for the efficacy of the licensed anti-VEFG drugs for nAMD, set avoidance of moderate visual loss, i.e. losing fewer than 15 ETDRS letters from the baseline at 12 months as their primary endpoint $(16,17)$. Our findings indicate that non-refracting VA which is routinely measured by a variety of HCPs in busy AMD clinics may be inadequate in detecting either of the two endpoints given the wide limit of agreement with the BCVA, between 18.39 and - 14.66 ETDRS letters - a range of 33 letters on the ETDRS chart. The non-refracting HCP measured VA showed higher inaccuracy and imprecision at lower levels of BCVA. 
Variation between two VA measurements has been reported for the same eye tested by the same examiner, using the same chart, even when the VA was measured within a one-minute interval (18). Despite a likely memorisation effect there was still a difference of \pm 2.5 letters between the two measurements on ETDRS vision chart (18). However, in a previous report in a group of patients from the Macular Photocoagulation Study, the BCVA measurement of patients by two certified clinical trial examiners on the same day found a mean difference of -0.75 letters with a standard deviation of 4.25 letters, and a limit of agreement between -9.08 and +7.58 letters on ETDRS vision chart (19). Another study also demonstrated considerable intersession variability in BCVA measurement among AMD patients, although measurements were not taken on the same day (20).

A consequence of inaccurate and imprecise VA measurements is eligibility to funded treatment of licensed drugs for ophthalmic conditions. Around half of the eyes with nAMD in our cohort met NICE stipulated VA cut-offs to qualify for the licensed drugs $(7,8)$. Compared to BCVA, the VA measured by the non-refracting HCP missed about $10 \%$ of patients who did qualify for the nAMD licensed drugs, whilst it included $15 \%$ of patients who didn't.

VA outcomes discrepancies between the 'real world' and clinical trials, as well as variation between different health systems or centres within the same health system, have been highlighted (12). Accuracy of the "real world" clinic measured VA may be a contributing factor to variation in reported nAMD treatment outcomes when differences cannot be explained otherwise.

Finally, eligibility for visual impairment registration and driving may be affected by accuracy and precision of VA measurements. In this study, when refracted BCVA was carried out, $60 \%$ of the eyes had a visual acuity below the UK Driver and Vehicle Licensing Agency (DVLA) driving standard for a car (11). The non-refracting HCP measured VA missed $4 \%$ of these eyes, while erroneously including about $10 \%$ of eyes that had met driving standards. Although we did not measure binocular vision as required by the DVLA, from the inaccurate VA measurement by HCPs, we can extrapolate that these findings could have serious legal implications.

The strength of this study is that it is a well-powered study for the relevant clinical end points. The study compares a typical clinical setting to the reference standard used in clinical trials.

The main weakness of this work is that it is a single centre study. Different centres will have different training protocols and quality assurance for the non-refracting HCPs measuring VA. Furthermore, it would have been helpful to include a cohort of measurements comparing optometrists measuring VA without and with subjective refraction in order to remove the influence of academic background and training when comparing the two methods. This was logistically beyond our resources.

The non-refracting HCPs were aware that this study was being conducted. Due to the Hawthorne effect, we expect an improvement in their performance in measuring visual acuity. 
Although there was an obvious difference in technique, non-refracting vs refracting VA, there could be other factors in play. One could speculate on the technique and the training of the non-refracting HCPs in a busy AMD clinic explaining the difference in VA. For instance, due to time constraints, patients with unstable fixation or a scotoma may not be encouraged to read, guessing where necessary, every single letter on each line of the ETDRS chart. Also, moving the vision chart from 4 metres to 1 metre, for the $4 \mathrm{~m}$ ETDRS charts when the top line cannot be fully read, could have been considered a time-consuming practice and not uniformly followed. As a result, low visual acuity levels would not be correctly measured, which is suggested by the low sensitivity of clinic measured VA vs BCVA in detecting VA of $<24$ letters (Table 3). However, the difference in the VA could be due to the inherent variation in non-refracted VA. To investigate these issues further, appropriate quality assurance procedure needs to be followed and the non-refracting HCPs have to be supported and trained if needed.

The UK does not have a certification system for non-refracting VA assessors. There are local protocols that departments may resort to in order to train their staff. However, an unstructured training coupled with the need to quickly deploy non-refracting VA assessors who have the most diverse knowledge and background lends itself to variation. Under pressure to process as many patients as possible assessors may adapt their VA measurement strategy. We cannot comment on the effect of variation of local standard operational procedures on VA measurements as this was beyond the scope of a single centre service evaluation study. We are highlighting that, potentially, there is an issue with "real world" VA measurements in the NHS. In a national health service that strives to collect VA data for monitoring outcomes, efforts should be made to achieve consistency and quality. This report may encourage revision of the standard operating procedure for VA measurement in ophthalmology departments, promote audits and inform a discussion on whether training national standards and quality assurance processes may be required to achieve a more robust and reproducible measurement of the most important assessment of visual function.

\section{Conclusions}

We found non-refracting HCP measured VA in eyes with nAMD to have limitations. The non-refracting HCP measured VA showed less accuracy and precision than refracted BCVA by a certified optometrist with a limit of agreement of 33 letters. This has potential implications on patient eligibility to licensed treatments, translation of clinical trial results into clinical practice, evaluation of treatment response and outcomes, certification of visual impairment and eligibility to driving for nAMD patients managed in eye clinics in the UK.

\section{Declarations}

Ethics approval and consent to participate:

This work was carried out as part of service evaluation which was formally approved by the hospital clinical governance and research, innovation \& development office.As such, research ethical approval was 
not required. Patients were informed of the study and verbal consent was obtained. The study was conducted in accordance with the declaration of Helsinki.

Consent for publication:

Not applicable.

Availability of data and materials:

The datasets used and/or analysed during the current study are available from the corresponding author on reasonable request.

Competing interests:

We have read and understood the BMC Ophthalmology policy on competing interests and declare the following interests: SP is paid advisor to Heidelberg Engineering ${ }^{\circledR}$, Zeiss $\AA$, Novartis $\AA$, Bayer ${ }^{\circledR}$ and Allergan ${ }^{\circledR}$.

Funding:

None.

Author's contributions:

OK designed the study, analysed the data, and wrote the manuscript. AC helped design the study, collected the data, and critically appraised the manuscript. SP helped design the study and critically appraised the manuscript.

Acknowledgements:

Authors would like to thank the staff of the Department of Ophthalmology, University Hospitals Coventry and Warwickshire, Coventry, United Kingdom for their help. This research received no specific grant from any funding agency in the public, commercial, or not-for-profit sectors.

\section{References}

1. Kniestedt C, Stamper RL. Visual acuity and its measurement. Ophthalmol Clin North Am. 2003;16(2):155-70.

2. Colquitt JL, Jones J, Tan SC, Takeda A, Clegg AJ, Price A. Ranibizumab and pegaptanib for the treatment of age-related macular degeneration: a systematic review and economic evaluation. Health Technol Assess. 2008;12(16):iii-iv, ix-201.

3. Owen CG, Jarrar Z, Wormald R, Cook DG, Fletcher AE, Rudnicka AR. The estimated prevalence and incidence of late stage age related macular degeneration in the UK. Br J Ophthalmol. 2012;96(5):7526. 
4. Bunce $C$, Wormald R. Leading causes of certification for blindness and partial sight in England \& Wales. BMC Public Health. 2006;8(58).

5. Lim LS, Mitchell P, Seddon JM, Holz FG, Wong TY. Age-related macular degeneration. The Lancet. 2012;379(9827):1728-38.

6. Solomon SD, Lindsley K, Vedula SS, Krzystolik MG, Hawkins BS. Anti-vascular endothelial growth factor for neovascular age-related macular degeneration. Cochrane Database of Systematic Reviews. 2019(3).

7. NICE. Ranibizumab and pegaptanib for the treatment of age-related macular degeneration: National Institute for Care Excellence; 2008 [updated 01/05/2012. Available from: https://www.nice.org.uk/guidance/ta155/chapter/1-Guidance.

8. NICE. Aflibercept solution for injection for treating wet age-related macular degeneration: National Institute for Care Excellence; 2013 [Available from: https://www.nice.org.uk/guidance/ta294/chapter/1-Guidance.

9. Amoaku WM, Chakravarthy U, Gale R, Gavin M, Ghanchi F, Gibson J, et al. Defining response to antiVEGF therapies in neovascular AMD. Eye. 2015;29(6):721-31.

10. DoH. Certificate of Vision Impairment: Explanatory Notes for Consultant Ophthalmologists and Hospital Eye Clinic Staff in England In: Health Do, editor. 2016.

11. DVLA. Visual disorders: assessing fitness to drive: Advice for medical professionals to follow when assessing drivers with visual disorders.: Driver and Vehicle Licensing Agency; 2016 [updated 4/03/2020. Available from: https://www.gov.uk/guidance/visual-disorders-assessing-fitness-to-drive.

12. Liew G, Lee AY, Zarranz-Ventura J, Stratton I, Bunce C, Chakravarthy U, et al. The UK Neovascular AMD Database Report 3: inter-centre variation in visual acuity outcomes and establishing real-world measures of care. Eye. 2016;30(11):1462-8.

13. Ferris FL, III, Kassoff A, Bresnick GH, Bailey I. New Visual Acuity Charts for Clinical Research. American Journal of Ophthalmology. 1982;94(1):91-6.

14. Faul F, Erdfelder E, Lang A-G, Buchner A. G*Power 3: A flexible statistical power analysis program for the social, behavioral, and biomedical sciences. Behavior Research Methods. 2007;39(2):175-91.

15. Altman DG, Bland JM. Measurement in Medicine: the Analysis of Method Comparison Studies. The Statistician. 1983;32(3):307-17.

16. Rosenfeld PJ, Brown DM, Heier JS, Boyer DS, Kaiser PK, Chung CY, et al. Ranibizumab for Neovascular Age-Related Macular Degeneration. New England Journal of Medicine. 2006;355(14):1419-31.

17. Heier JS, Brown DM, Chong V, Korobelnik J-F, Kaiser PK, Nguyen QD, et al. Intravitreal Aflibercept (VEGF Trap-Eye) in Wet Age-related Macular Degeneration. Ophthalmology. 2012;119(12):2537-48.

18. McMonnies CW. Chart memory and visual acuity measurement. Clin Exp Optom. 2001;84(1):26-33.

19. Blackhurst DW, Maguire MG. Reproducibility of refraction and visual acuity measurement under a standard protocol. The Macular Photocoagulation Study Group. Retina. 1989;9(3):163-9. 
20. Patel PJ, Chen FK, Rubin GS, Tufail A. Intersession Repeatability of Visual Acuity Scores in AgeRelated Macular Degeneration. Investigative Ophthalmology \& Visual Science. 2008;49(10):4347-52.

Figures

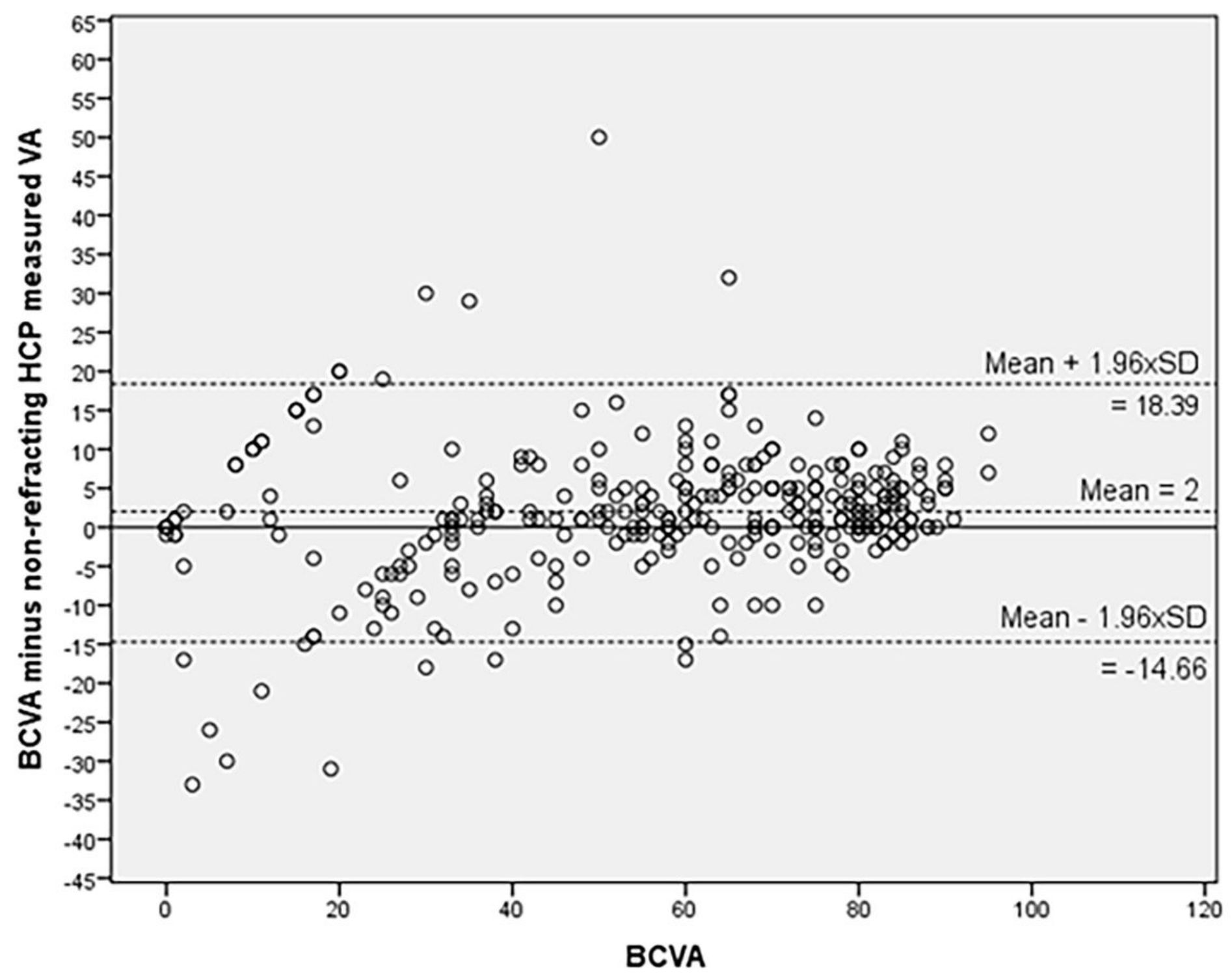

Figure 1

The Bland-Altman plot showing the relationship between BCVA and the BCVA minus non-refracting HCP measured VA. The difference between BCVA and non-refracting HCP measured VA has a limits of agreementbetween 18.39 ETDRS letters and -14.66 ETDRS letters. 